\title{
TRIM8 downregulation in glioma affects cell proliferation and it is associated with patients survival
}

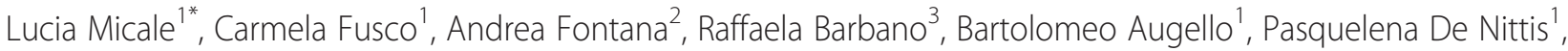
Massimiliano Copetti ${ }^{2}$, Maria Teresa Pellico ${ }^{1}$, Barbara Mandriani ${ }^{1}$, Dario Cocciadiferro ${ }^{1,4}$, Paola Parrella ${ }^{3}$, Vito Michele Fazio ${ }^{3}$, Lucia Maria Cecilia Dimitri ${ }^{5}$, Vincenzo D’Angelo ${ }^{6}$, Chiara Novielli', Lidia Larizza ${ }^{7,8}$, Antonio Daga ${ }^{9}$ and Giuseppe Merla ${ }^{1 *}$

\begin{abstract}
Background: Human gliomas are a heterogeneous group of primary malignant brain tumors whose molecular pathogenesis is not yet solved. In this regard, a major research effort has been directed at identifying novel specific glioma-associated genes. Here, we investigated the effect of TRIM8 gene in glioma.

Methods: TRIM8 transcriptional level was profiled in our own glioma cases collection by qPCR and confirmed in the independent TCGA glioma cohort. The association between TRIM8 expression and Overall Survival and Progressionfree Survival in TCGA cohort was determined by using uni-multivariable Cox regression analysis. The effect of TRIM8 on patient glioma cell proliferation was evaluated by performing MTT and clonogenic assays. The mechanisms causing the reduction of TRIM8 expression were explored by using QPCR and in vitro assays.

Results: We showed that TRIM8 expression correlates with unfavorable clinical outcome in glioma patients. We found that a restored TRIM8 expression induced a significant reduction of clonogenic potential in U87MG and patient's glioblastoma cells. Finally we provide experimental evidences showing that miR-17 directly targets the 3' UTR of TRIM8 and post-transcriptionally represses the expression of TRIM8.

Conclusions: Our study provides evidences that TRIM8 may participate in the carcinogenesis and progression of glioma and that the transcriptional repression of TRIM8 might have potential value for predicting poor prognosis in glioma patients.
\end{abstract}

Keywords: TRIM8, Glioblastoma, miR-17, Cell proliferation

\section{Background}

Human gliomas are a heterogeneous group of primary malignant brain tumors, which most commonly occur in central nervous system of both children and adults [1]. Glioblastoma multiforme (GBM), the most aggressive form of glioma, exhibits advanced features of malignancy, such as rapid tumor cell proliferation, intense apoptosis resistance, florid necrosis, and robust angiogenesis [2]. The tumor properties underlie the poor clinical outcome by conferring strong resistance to chemotherapy and

\footnotetext{
*Correspondence: I.micale@operapadrepio.it; g.merla@operapadrepio.it 'Medical Genetics Unit, IRCCS Casa Sollievo della Sofferenza, Poliambulatorio Giovanni Paolo II, I-71013 San Giovanni Rotondo (FG), Italy

Full list of author information is available at the end of the article
}

radiotherapy, and by promoting a neurologically debilitating course leading to death within 12-18 months post diagnosis [3]. TRIM8 maps to chromosome 10q24.3, a region showing frequent deletion and loss of heterozygosity in human glioma [4]. TRIM8 encodes a member of the tripartite-motif-containing (TRIM) protein super family involved in a broad range of biological processes, including carcinogenesis [5]. TRIM8 interacts with and negatively regulates PIAS3, a protein inhibitor of IL-6dependent activation of STAT3, a signaling pathway important for cancer development and progression [6]. In agreement with previously data, we recently reported TRIM8 as a new modulator of the p53-mediated tumor suppression mechanism [7]. Under stress conditions, such

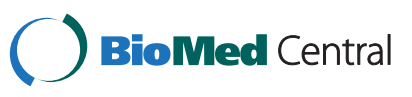


as UV exposure, we showed that p53 induces the expression of TRIM8, which in turn stabilizes p53 leading to cell cycle arrest and reduction of cell proliferation through enhancement of p21 and GADD45 expression [7]. Experimental evidence has outlined TRIM8 as one of the genes which low expression level correlates with nodal metastatic progression in primary larynx squamous cell carcinoma and whose expression inhibits tumor cell colony formation in vitro [8]. Finally, TRIM8 deficit has been showed to impair p53-mediated cellular responses to chemotherapeutic drugs in a model of Renal Cell Carcinoma [9]. Up regulation of $m i R-17$, associated with advanced tumor progression and poor overall survival of gliomas [10], has been shown to reduce the levels of TRIM8 in primary chronic lymphocytic leukemia cells, although a direct regulation was not yet demonstrated [11].

In this study, we showed that TRIM8 is down regulated in glioma tissues and cell lines and its expression inversely correlates with tumor grade. We found that a restored TRIM8 expression in patient glioma cell lines suppresses the tumor growth and induced a significant reduction of clonogenic potential. Finally we showed that miR-17 directly targets the 3'UTR of TRIM8 and post-transcriptionally represses the expression of TRIM8.

\section{Methods}

\section{Patients and samples}

In this study we collected 70 specimens at the time of surgery from the Neurosurgery Unit IRCCS, Casa Sollievo della Sofferenza (CSS), San Giovanni Rotondo, Italy. The Ethic Committees of the IRCCS, CSS, approved this study. Prior written and informed consent was obtained from each patient in accordance with Institution Guidelines and acceptance. Upon receipt from surgery, one half of the tissue sample from the bulk of the tumor was immediately frozen in liquid nitrogen and stored at $-80{ }^{\circ} \mathrm{C}$. The second half was processed for primary tumor cultures. After surgery, glioblastoma patients were treated according to the Stupp protocol [12]. All cases were newly diagnosed gliomas, pathologically classified according to the WHO system. Clinical-pathological patients' characteristics are reported in Additional file 1: Table S1. The median follow up time along with interquartile range (IQR) was 17.8 (IQR: 10.7-27.7) months.

\section{Primary tumor cell cultures generation}

Glioma samples were mechanically disassociated into single cells using sterile scalpels. Tumor short-term cells suspension was cultured in a poly-lysine-coated T25 flask at $37{ }^{\circ} \mathrm{C}$ and $5 \% \mathrm{CO} 2$ and expanded in DMEM/ F12 medium containing $10 \%$ foetal calf serum, $1 \%$ penicillin/streptomycin. Human U87MG glioma cell lines were grown following standard protocols. All cell culture media were purchased from Life Technologies.
We were successful in obtaining primary cell culture for 45 glioma patients. Additional RNAs and DNAs from glioma-derived cell lines were reported in [13] and provided by Dr. Daga, IST, Genova. Normal human astrocyte (NHA) cell line was gently provided by Dr. Fanelli Mirko, University of Urbin, Italy.

\section{Quantitative real time reverse transcription-PCR (qPCR)}

Total RNA was extracted using TRIZOL reagent (Life Technologies). Integrity and purity of RNAs were measured by using Agilent 2100 Bioanalyzer (Agilent Technologies) and reverse-transcribed by Quantitect Transcription kit (Qiagen), according to the manufacturer's instructions.

Oligos for qPCR were designed using the Primer express program [14] with default parameters, with EEF1A1 and $T B P$ as references genes. qPCR reactions and calculations were made as reported in $[15,16]$, mRNA from NHA cell line was used as reference sample for cell line, while commercially available RNAs (Agilent Technologies) from brain of 4 healthy individuals were used in tissues gene expression studies, respectively.

\section{Mutational Analysis and copy number variation analysis} Genomic DNAs were extracted from fresh and frozen peripheral blood leukocytes and from cell lines using an automated DNA extractor (EZ1, Qiagen) and quantified by Nanodrop (Thermo Scientific). Sequencing of TRIM8 coding region was performed in 70 patients. Primers were designed using the Primer 3 Output program (http://frodo.wi.mit.edu/primer3/) to amplify the 6 coding exons of TRIM8 (RefSeq NM_030912.2) gene including the intronic flanking sequences. The amplified products were subsequently purified and sequenced as reported in [17]. All primers used in this study are available upon request. For TRIM8 gene copy number variation analysis, four normalization assays mapping to HSA21 and four normalization DNAs were systematically included in each run as described [16]. Gene dosage segments were classified as chromosomal "gain" or "loss" if the absolute value of the predicted dosage was more than 0.75 times the interquartile range of the difference between observed and predicted values for each region. Primer pairs were designed to amplify a fragment spanning the codon 132 that encodes for the catalytic domain of IDH1. Sequence reactions were performed as reported in [17].

\section{miRNA expression analysis}

A qPCR for miR-17 expression in glioma cell lines and tissues was performed using TaqMan miRNA Reverse Transcription kit according to the manufacturer's instructions. Reactions were set up in a 384-well plate using Taqman Universal PCR Master Mix (Life Technology) and run in an ABI Prism7900HT according to the manufacturer's 
instructions. All qPCR experiments were performed in triplicate and the small nucleolar RNA U6 expression level was used as an endogenous control. All qPCR reagents were purchased from Life Technologies.

\section{Dual-luciferase reporter assay and constructs}

The entire genomic region of the miR-17-92 cluster (1094 bp) was amplified by using gene-specific primers and cloned into pcDNA3 expression vector (pcDNA3miR-17-92). The luciferase-UTR reporter plasmid was constructed by introducing the TRIM8 3'-UTR into pmiR-REPORT miRNA Expression Reporter Vector System (Life Technologies). The TRIM8 3'-UTR sequence was amplified by PCR from HeLa cDNA. Mutagenesis was used to delete miR-17 binding site using the QuickChange II kit (Stratagene). The pcDNA3-Flag-TRIM8-3' UTR was generated by cloning the entire open reading frame and 3'-UTR of TRIM8 amplified from HeLa cDNA into pcDNA3-Flag vector. All constructs were verified by sequencing. The reporter construct, pSV-Renilla (pRLSV40, Promega) and miR-17 mimic (or miR-17-5p hairpin inhibitor or miR-20a mimic, Dharmacon) or pcDNA3miR-17-92 were transfected into H1299, HEK293 or MCF-7 cells using Hyperfect Transfection Reagent (Qiagen) or Lipofectamine 2000 (Life Technologies). After $48 \mathrm{~h}$, the cells were lysed in passive lysis buffer and assayed for both firefly and renilla luciferase activity using the Dual-GLO ${ }^{\circ}$ Luciferase Assay System (Promega). Firefly luciferase activity was normalized to Renilla luciferase activity for each transfected well. Values are the mean \pm S.E.M. of three experimental replicates from two to four independent transfections. Significance was determined by a two-tailed unpaired $t$ test for means.

\section{MTT cell proliferation assay}

Tumor cells were transfected with pcDNA3-myc-TRIM8 or empty vector using Lipofectamine LTX (Life Technologies) according to the manufacturer's instructions and 24, 48 or $72 \mathrm{~h}$ after transfection MTT (3-2, 5-diphenyl tetrazolium bromide, Sigma) was added to each well of a culture plate as described previously [7]. After incubation at $37^{\circ} \mathrm{C}$ for $4 \mathrm{~h}$, the reaction was stopped by solubilization with isopropanol. Absorbance at a wavelength of $570 \mathrm{~nm}$ was measured. Each experiment was performed in triplicate.

\section{Clonogenic assay}

Clonogenic assay was performed as previously described [18]. In brief, $5 \times 10 \mathrm{e} 5$ U87MG and three primary glioblastoma cells were transfected with 5 micrograms of pcDNA3 vector coding for HA-TRIM8 or empty vector by using Neon Transfection Device (Life Technologies). $48 \mathrm{~h}$ post electroporation, the cells were analyzed for HA expression through immunofluorescence and plated in 96-well plates with a density of 0.8 cell/well. After 3 weeks, colonies defined as greater than 50 cells were counted. Each set of experiments was performed in triplicate.

\section{TCGA mRNA, miRNA dataset and patients' information} mRNA, miRNA expression data and clinical information for the Glioblastoma Multiforme and Lower Grade Gliomas (LGG) datasets were downloaded from The Cancer Genome Atlas (TCGA) data portal in January and June 2013 respectively (http://cancergenome.nih.gov/) [19]. We analysed level 3 data for a total of 945 patients (567 glioblastoma and 378 LGG) with TRIM8 expression and tumor grade information available. Data was quartile normalized and $\log 2$ transformed. Among 378 patients with LGG, 183 had WHO grade II, 195 had WHO grade III. To perform overall survival (OS) and progression-free survival (PFS) analyses, we selected 935 patients (564 glioblastoma and 371 LGG) from the 945 patients, with both outcome available information miRNA-based subtype classification. Since grade II $(\mathrm{n}=180$, from the 935 patients $)$ and grade III ( $\mathrm{n}=191$, from the 935 patients) gliomas differ substantially in prognosis, survival analyses were performed in each subgroup separately. To evaluate association between TRIM8 CNV and tumor grade, a total of 526 patients (from the 945) with TRIM8 CNV information available were selected (268 glioblastoma and 258 LGG). Clinical-pathological patients' characteristics for the TCGA GBM and LGG datasets are reported in Additional file 2: Table S2.

\section{Statistical methods}

Continuous variables were reported as mean \pm standard deviation (SD) and as median along with IQR. Categorical variables were reported as absolute and relative frequencies. Normal distribution assumption was checked by means of Q-Q plot, Shapiro-Wilks and KolmogorovSmirnov tests.

Due to the deviation from normality distribution assumption, all statistical analyses were performed using the log-transformed expressions for TRIM8 in glioma cell lines and tissues, whereas the square root transformation was applied for $m i R-17-5 p$ expression levels only.

Differences of TRIM8 in glioma tissues expression between tumour grades (WHO grades II vs. III vs. IV) were assessed using ANOVA model.

Histograms of TRIM8 copy numbers frequency distributions were further represented with respect to tumor grades. The linear trend of TRIM8 copy numbers across tumor grades was tested using Mantel-Haenszel ChiSquare. Boxplots of TRIM8 expression in tissue gliomas with respect to each tumor grade and TRIM8 copy number available were also reported. Time-to-event analyses were performed by univariable and multivariable Cox 
proportional hazards regression models for PFS and OS outcomes and the tertile values of TRIM8 expression were used to categorize patients into lowest, medium and highest expression groups. Risks were reported as hazards ratios (HR) along with their $95 \%$ confidence interval $(95 \% \mathrm{CI})$. Multivariable Cox proportional hazard models included the following covariates: TRIM8 expression (in tertiles), age, the presence of $I D H 1$ mutation and the presence of any treatment therapy (i.e. Chemotherapy, Radiotheraphy or both). Time to disease progression was defined as the time between diagnosis and first evidence of disease progression, whereas time to death was defined as the time between diagnosis and death occurrence. Furthermore, Kaplan-Meier curves were also reported along with p-value from log-rank test. A p-value $<0.05$ was considered statistically significant. All statistical analyses and graphs were performed by using SAS Release 9.3 (SAS Institute, Cary, NC, USA) and $\mathrm{R}$ (version 2.15.2, packages: gtools, car, survival) software, respectively.

\section{Results}

TRIM8 is down regulated in higher-grade gliomas

TRIM8 RNA expression level was determined from 71 primary glioma cell lines: 8 (WHO grade I and II), 12 (III), 51 (IV) and 70 tumor tissues: 16 (WHO grade I and II), 10 (III), 44 (IV). TRIM8 relative expression ranged from 0.09 to 1.65 in glioma cell lines and 0.01-16.13 in tumor tissues, respectively. We found a statistically significant lower TRIM8 relative expression in grade IV, when compared with grades II and III $(\mathrm{p}<0.001)$ in both tumor tissues and cell lines (Fig. 1a and b). In tumor tissue glioma, median TRIM8 relative expressions were 0.49 in GBM (IQR: 0.30-0.82), 0.81 in WHO grade III (IQR: 0.37-2.32) and 2.61 in WHO grade I-II (IQR:1.52-6.14) glioma (Fig. 1a). We next examined the transcriptional profile of TRIM8 in an independent cohort, i.e. the TCGA cohort, detecting a significant lower expression of TRIM8 in grade III glioma (Median:0.65, IQR:0.32-1.23) as compared with grade II glioma (Median: 1.05, IQR 0.63-1.79) (Fig. 1c). GBM expression profile data were not analyzed and compared as they were generated by a different platform compared to LGG (https://tcga-data.nci.nih.gov/tcga/).

\section{Low TRIM8 tissues expression level is associated with unfavorable clinical outcome in WHO grade III gliomas}

We evaluated the association between TRIM8 expression and Overall Survival and Progression-free Survival in 180 grade II, 191 grade III and 564 grade IV gliomas of TCGA cohort. Tertiles of TRIM8 expression were calculated within each tumor grade, separately.

In WHO grade III tumors, univariable Cox regression analysis revealed a significant increase of mortality risk in patients with the lowest TRIM8 expression levels $(<0.45$, first tertile) as compared to those with the highest levels ( $>1.00$, third tertile). Indeed, we estimated a HR of 0.09 (95\% CI: 0.04-0.22, p<0.001) and 0.18 (95\% CI: 0.08$0.36, \mathrm{p}<0.001)$ comparing the third and the second tertiles with the first one, respectively. Such results were confirmed in the multivariable Cox regression analysis comparing the third tertile to the first tertile ( $\mathrm{HR}=0.23$, 95 \% CI: 0.10-0.54, p= 0.001) (Fig. 1d). Moreover, univariable Cox analysis also evidenced a significant increase in the risk of disease progression in patients with the lowest TRIM8 expression levels with respect to those with the highest level (third vs. first tertiles $\mathrm{HR}=0.20,95 \% \mathrm{CI}$ : 0.05-0.76, $\mathrm{p}=0.018$; second vs. first tertiles $\mathrm{HR}=0.43$; $95 \%$ CI: 0.15-1.22, $\mathrm{p}=0.114$ ) (Fig. 1e). No significant differences were found among tertiles group in multivariable analysis, probably due to the low number of progression events, with a consequent loss of statistical power. Overall these data suggested that TRIM8 expression levels might represent an independent predictor of survival in WHO grade III gliomas. No statistically significant association with OS and PFS were found in WHO grade II gliomas and GBM.

\section{TRIM8 reduces cell proliferation in glioblastomas}

To evaluate the effect of TRIM8 in glioma cell proliferation, we transfected U87MG glioma cells with a vector expressing TRIM8 and measured cell viability by MTT assay at 24,48 and $72 \mathrm{~h}$ post transfection, respectively. Results showed that TRIM8 overexpression significantly reduced cell proliferation by about $25 \%$ (Fig. 1f, $\mathrm{p}<0.05$, Additional file 3: Figure S1A). We next analyzed the biological effect of TRIM8 expression by restoring expression levels of TRIM8 in two representative GBM patients cell lines. MTT assay confirmed that, in the presence of TRIM8, the rate of cell proliferation is significantly reduced by about $30 \%$ (Fig. 1g, p < 0.01).

We therefore investigated whether overexpression of TRIM8 affects clonogenic potential of glioma cells as an indirect index of their tumorigenic potential. U87MG glioma cells, transfected with expressing TRIM8 or control vector (with transfection efficiency $>80 \%$, measured by immunofluorescence, data not shown), were plated at limiting dilution and the formation of large colonies ( $>50$ cells) was assessed after 3 weeks. The enforced expression of TRIM8 induced a significant reduction of clonogenic potential $(\mathrm{p}=$ 0.014) from the average number of $47.9 \%$ colonies in control-transfected cultures to $30.4 \%$ in TRIM8 transfected cells (Fig. 1h). Consistently these results were confirmed in three primary glioblastoma cell lines (GBM3, GBM6, and GBM19) with an average number of $55 \%, 49.3 \%$, and $42.5 \%$ colonies in control cultures, respectively, and $35.3 \%$. $39.3 \%$, and $28.9 \%$, respectively, in glioma TRIM8 expressing cells (Fig. 1j, Additional file 3: Figure S1B). 
A

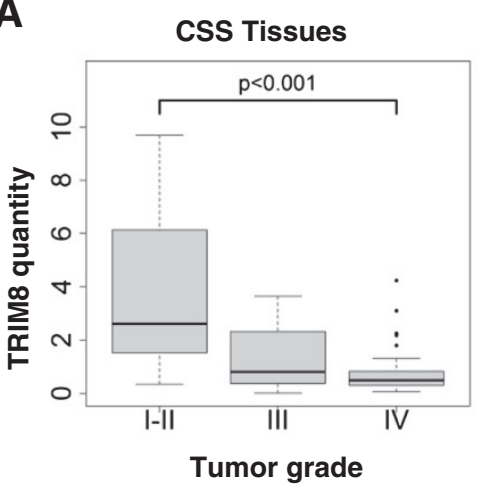

D

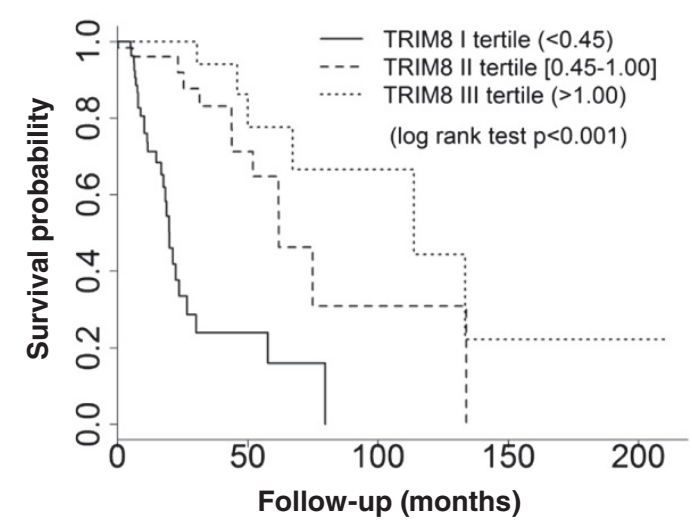

F

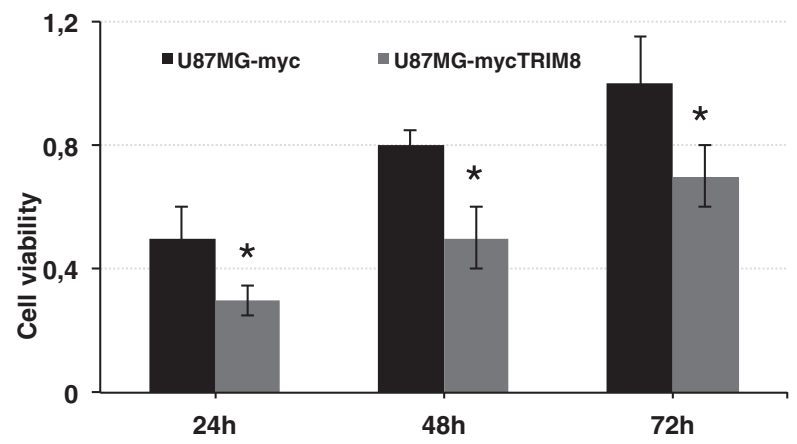

H

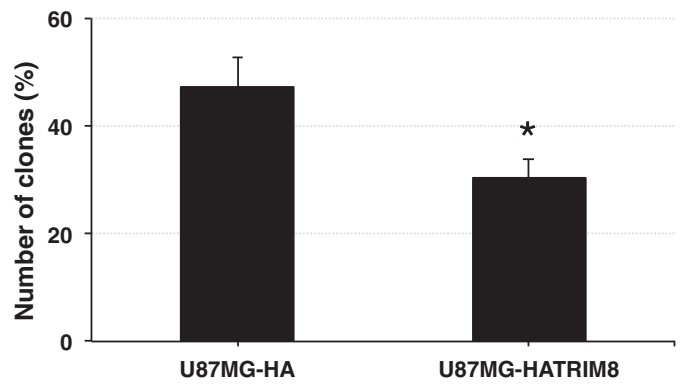

E
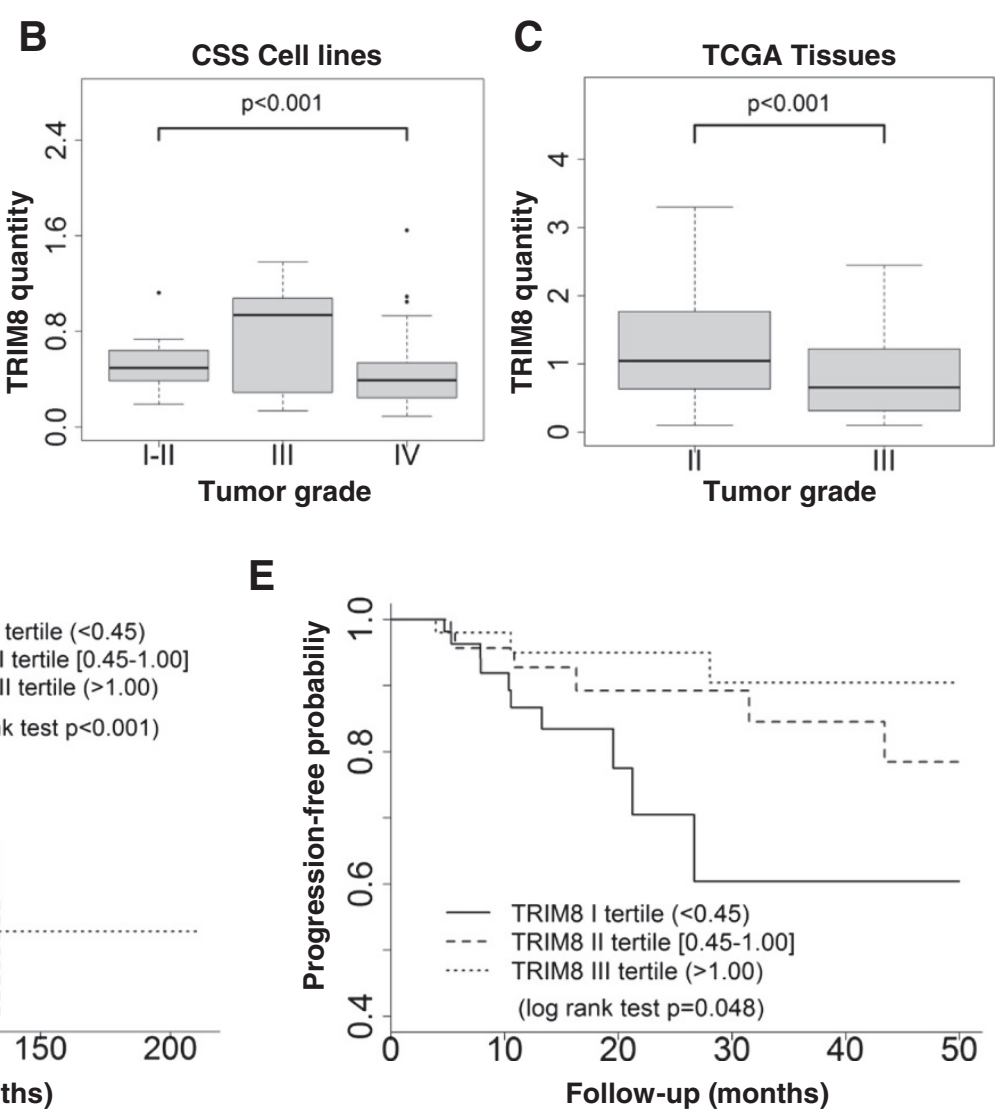

G

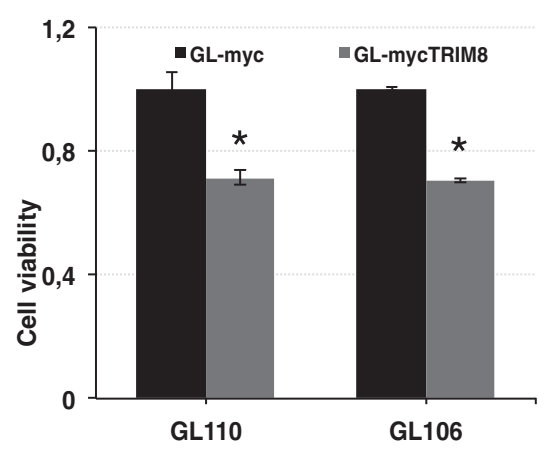

J

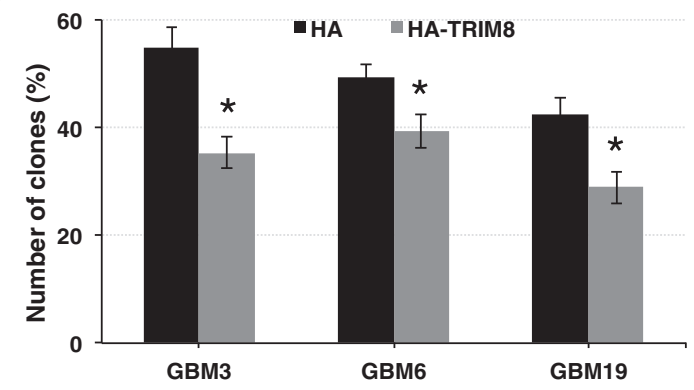

Fig. 1 (See legend on next page.) 
(See figure on previous page.)

Fig. 1 TRIM8 is downregulated in gliomas and affects cell proliferation. (a-c) Box-plots of TRIM8 tissues expression (a, c) and TRIM8 cells expression (b) between tumor grades. GPCR was performed to measure the level of TRIM8 transcript in a total of 70 glioma tissues and cell lines (CSS) and 378 glioma tissues specimens from TCGA LGG dataset. Kaplan-Meier curves for Overall Survival (d) and Progression-Free-Survival (e) according to TRIM8 tertiles of expression levels in patients with tumor grade III (TCGA data). (f-g) The effect of TRIM8 expression on cell proliferation was assessed by MTT assay in U87MG glioblastoma cell lines $(\mathbf{f})$ and in patient glioma cell lines $(\mathbf{g})$ transfected with pcDNA3 vector expressing TRIM8 or empty vector. The Y-axis represents the absorbance value. (h-j) Effect of HA-TRIM8 expression and empty vector on in vitro clonogenic potential of U87MG cells (h) and three patient glioblastoma cell lines, GBM3, GBM6, and GBM19 (j). Results are reported as mean percentage of clones from three independent experiments, and error bars represent standard deviations. These experiments were repeated three independent times and similar results were obtained each time

\section{Loss TRIM8 copy number in glioma}

To investigate the mechanisms that may cause the reduction of TRIM8 expression in glioma we sequenced the coding and untranslated regions of TRIM 8 in 70 patients detecting any likely pathogenic variant (data not shown). Then we sought to determine TRIM8 copy number by qPCR. TRIM8 copy number results were obtained in 68 out of the70 patients from out cohort. We detected a somatic heterozygous deletion encompassing the full TRIM8 gene in 27 out of 68 (39.7\%) analyzed glioma cells. We found a linear trend of TRIM8 copy number across tumor grades (Fig. $2 \mathrm{a}, \mathrm{p}=0.025$ ). Specifically, a heterozygous deletion of TRIM8 was observed in approximately $11.1 \%$ of gliomas of WHO grades I and II, $30.8 \%$ of grade III, and $47.8 \%$ of glioblastomas (Fig. 2a).

To confirm this evidence, we used the available TRIM 8 copy number information from the 526 of TCGA cohort, detecting a heterozygous deletion of TRIM8 gene in 303 out of $526(57.6 \%)$ analyzed glioma tissues. Specifically, the loss of one copy of TRIM8 gene was observed in approximately $9.9 \%$ of gliomas of grade II, $32.8 \%$ of grade III, and $91.8 \%$ of glioblastomas (Fig. $2 \mathrm{~b}$, $\mathrm{p}<0.001)$. Loss of TRIM8 copy number was found significantly associated to TRIM8 low expression level in TCGA group (Fig. 2c-e).

\section{miR-17 regulates TRIM8 expression at the post-transcriptional level}

We screened the 3'UTR of TRIM8 by bioinformatic tools to search for putative miRNAs binding sites. As a result, we found that the 3'UTR of TRIM8 contains two putative conserved $m i R-17$ and $m i R-20 a$ binding sites. To experimentally in vitro test whether TRIM8 is directly targeted by $m i R-17$ and $m i R-20 a$, the 3'UTR of TRIM8 gene carrying wild type and mutated miRNA seed was inserted into a luciferase reporter vector and the entire genomic coding region of the $m i R-17-92$ cluster was cloned into pcDNA3 expression plasmid. We observed a luciferase reporter activity reduced by $60 \%$ in HEK293 cells co-transfected with the 3'UTR TRIM8 and miR-17-92 expressing vectors $(\mathrm{p}<0.05$, Fig. 3a).

Next, to test whether $m i R-17$ or $m i R-20 a$ directly target the 3'UTR of TRIM8, HEK293 cells were co-transfected with 3'UTR TRIM8 reporter construct along with a synthetic mimic of $m i R-17$ or of $m i R-20 a$. As shown in Fig. 3b the overexpression of $m i R-17$ significantly reduced the luciferase activity of the vector containing the 3'UTR of TRIM8 when compared to the control $(\mathrm{p}=0.005)$. Consistently, deletion of the binding site abrogated this effect (Fig. 3b). Collectively, these results indicated that the 3' UTR of TRIM8 is targeted by miR-17.

Further we explored whether this regulation occurred also for endogenous TRIM8. In keeping with our luciferase data, qPCR results showed a negative correlation between miR-17 and TRIM8 expression levels in several human cell lines including breast and glioma cells (Fig. 3c). On the other hand, MCF-7 cells transfected with $m i R-17$ inhibitor had a slight increased expression of TRIM8 mRNA when compared with miR control inhibitor (Fig. 3d). Finally, we found a slight negative correlation between TRIM8 and $m i R-17$ expression in glioma tissue in grade II $(\mathrm{r}=-0.172, \mathrm{p}=0.020)$ and glioblastoma $(\mathrm{r}=-0.088, \mathrm{p}=0.038)$ patients from TCGA cohort, supporting the assertion that $m i R-17$ might contribute to modulate TRIM8 expression in gliomas (data not shown).

In order to assess whether $m i R-17$ also regulates protein level of TRIM8 in glioma cells, we co-transfected U87MG cells with a pcDNA3-FLAG vector containing the ORF and 3'UTR of TRIM8 (hereafter named TRIM8-3'UTR), along with synthesized $m i R-17$ or $m i R-17$ inhibitor, respectively. The level of TRIM8-tagged fusion protein was reduced by $m i R-17$ mimic and enhanced by $m i R-17$ inhibitor (Fig. 3e). Overall these evidences demonstrate that $m i R-17$ regulates TRIM8 expression at both transcriptional and post-transcriptional level by directly binding the 3'UTR region of TRIM8.

\section{Discussion}

Human gliomas are the most common and lethal neurological malignancies in adults. Our study is the first that investigates the role of the E3 ubiquitin ligase TRIM8 in glioma. We presented experimental evidences showing that i) TRIM8 expression level is significantly decreased in glioma and is inversely associated with glioma WHO grades in both our own cases collection and in the independent TCGA glioma cohort, ii) lower TRIM8 tissues 

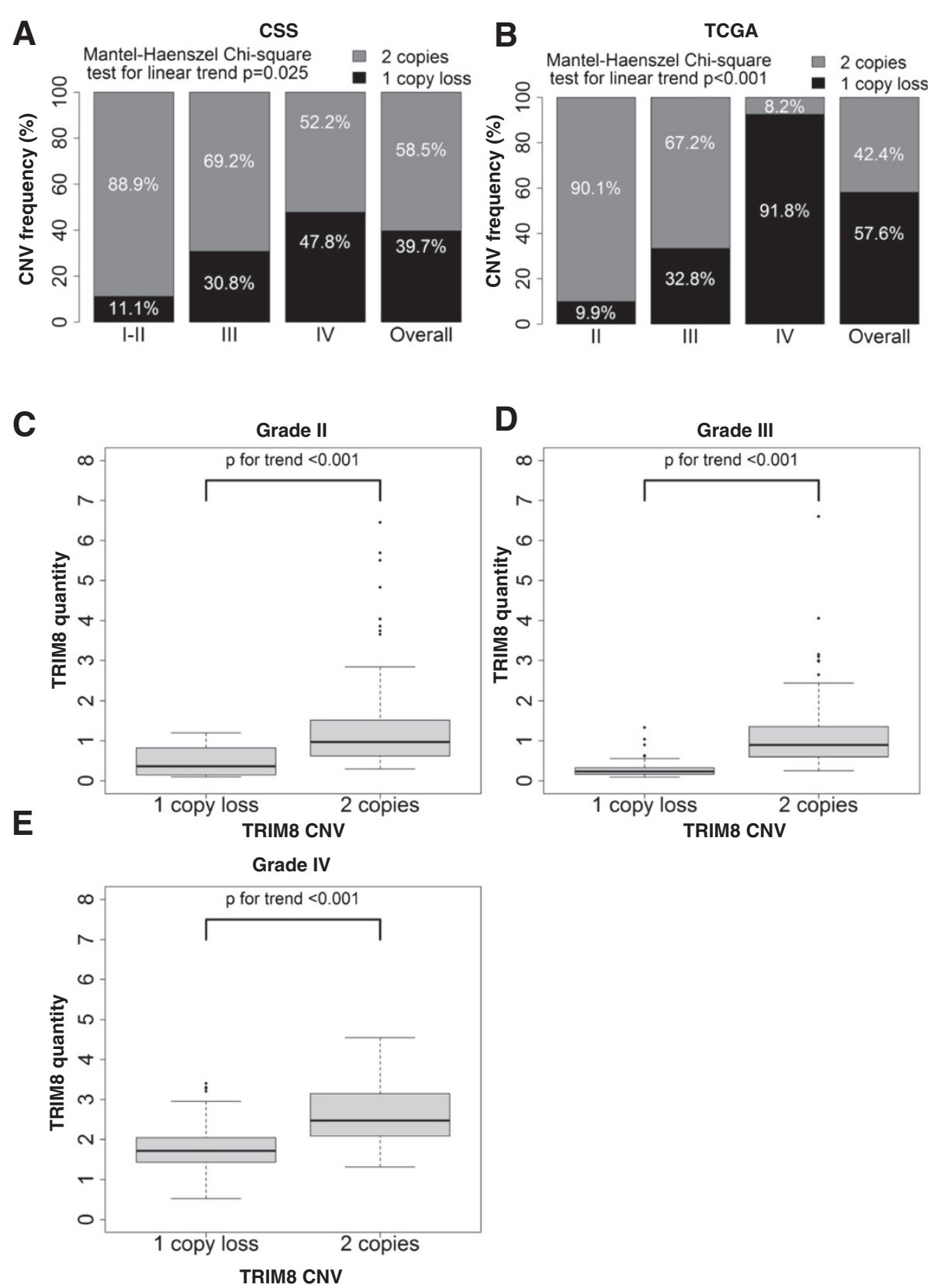

Fig. 2 Loss of TRIM8 copy number in gliomas. a Frequency distribution of TRIM8 copy number, within each tumor grade, along with p-value from Mantel-Haenszel Chi-Square test for linear trend. TRIM8 copy number in the gliomas was explored by using qPCR on DNA extracts from patient glioma cell lines and peripheral blood. b Frequency distribution of TRIM8 CNV by tumor grade (TCGA data). c-e Boxplots of TRIM8 expression in tissue gliomas by TRIM8 copy number for each tumor grade separately (TCGA data)

expression level is an independent predictor of risk of death in WHO grade III tumours (TCGA dataset), and iii) the overexpression of TRIM8 suppresses cell growth and induces a significant reduction of clonogenic potential in both U87MG glioblasto ma and patient's primary glioma cell lines.

We investigated the molecular mechanism that can explain the observed TRIM8 expression decrease. Our experimental data suggested that the inactivation of TRIM8 in glioblastoma cells may occurs primarily through the loss of gene copy number. Moreover we showed that TRIM8 expression is regulated by $m i R-17$ at transcriptional and post-transcriptional level. Accumulating data have indicated that the inhibition of miR-17 significantly reduce cell viability and increases apoptotic activity in glioma cell lines and the upregulation of this miRNA is associated with advanced tumour progression and poor overall survival of gliomas [20]. Our preliminary results suggest 


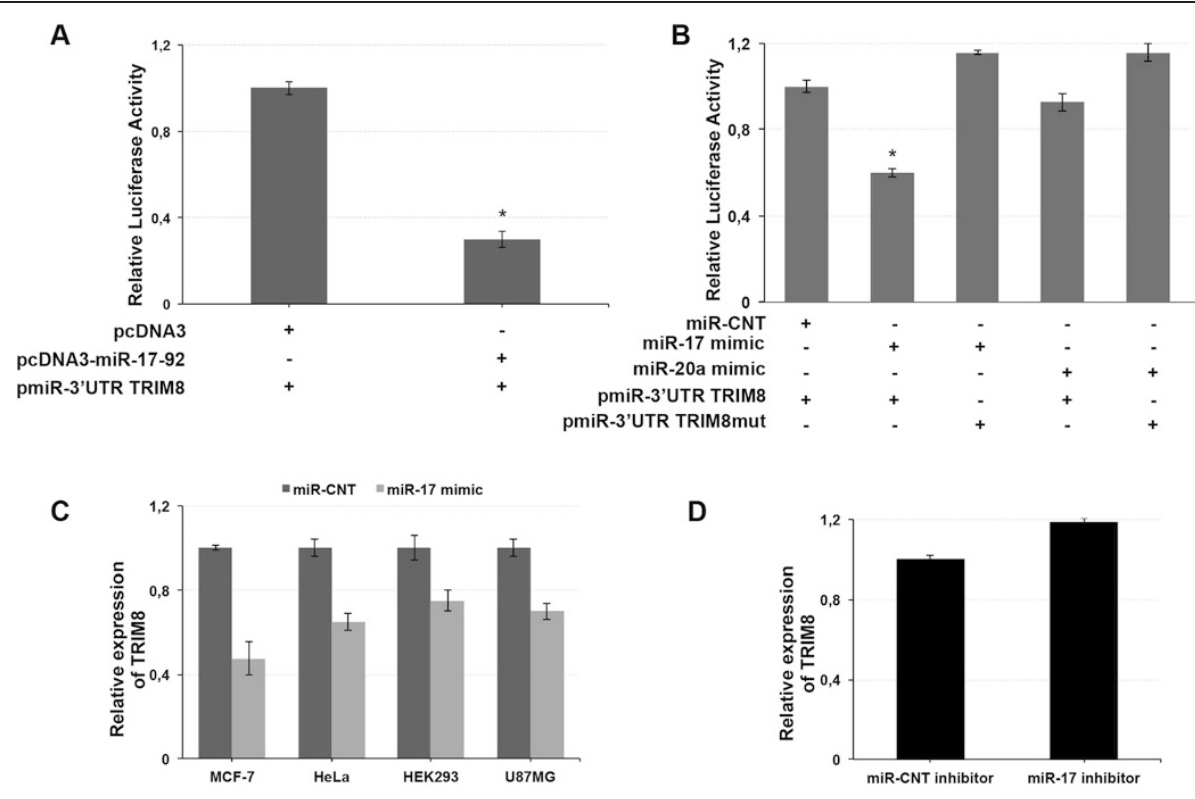

$\mathrm{E}$

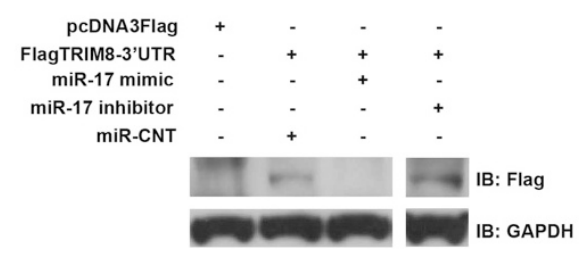

Fig. 3 miR-17 regulates TRIM8 expression at the post-transcriptional level. a HEK293 cells were co-transfected with a reporter construct carrying the 3' UTR of TRIM8 and a pcDNA3-miR-17-92 or pcDNA3 empty vector. Luciferase activities were measured and normalized to the level of control Renilla luciferase. b HEK293 cells were co-transfected with reporter constructs carrying the 3'UTR of TRIM8 or 3' UTR of TRIM8 containing mutated miR-17 complementary site and a synthetic mimic of miR-17, mimic of miR-20a, or miR-control (miR-CNT). c Detection of TRIM8 endogenous expression by qPCR in MCF-7, HeLa, HEK293, and U87MG cell lines transfected with miR-17 mimic or miR-control. d TRIM8 endogenous expression in MCF-7 cell lines transfected with miR-17 inhibitor or miR-control inhibitor. e Immunoblotting analysis by using indicated antibodies on whole protein lysate from U87MG transfected with a construct carrying the ORF and 3'UTR of TRIM8 or the ORF and 3' UTR of TRIM8 containing a deletion of miR-17 complementary site and a miR-17 mimic, miR-17 inhibitor, or miR-control

the existence of a feedback circuit involving miR-17 and TRIM8 for glioma pathogenesis.

Our data corroborate mounting evidence showing a downregulation of TRIM8 in different cancers and its role in controlling cell growth. In fact, recent data showed that TRIM8 low expression level correlates with nodal metastatic progression in primary larynx squamous cell carcinoma and whose expression inhibits tumour cell colony formation in vitro $[8,21]$. Yet, we previously ascertained that TRIM8 is an important component in controlling the molecular switch that directs p53 toward transcriptional activation of cell cycle arrest genes such as p21 and GADD45 [7]. Finally, downregulation of TRIM8 was shown to impair the p53-mediated cellular responses to chemotherapeutic drugs in renal cell carcinoma [9].

In our study, the analysis of the TCGA datasets evidenced a significant increase in the risk of death and disease progression in WHO grade III tumours with the lowest TRIM8 expression levels compared to those with the highest level. These results suggest that loss of TRIM8 expression may be necessary for the transition to a more aggressive phenotype typical of $\mathrm{WHO}$ grade III gliomas as compared with WHO grade II tumours, but it may have less effect on the clinical behaviour of GBMs, suggesting a likely role of TRIM8 gene in gliomagenesis and disease progression.

Many examples are showing that TRIM family members, through their E3 ubiquitin ligases activity, are involved in oncogenic processes such as cell proliferation and apoptosis [5, 22]. For instance, TRIM32 induces tumour necrosis factor (TNF)-mediated apoptosis through its direct interaction and ubiquitylation of X-linked inhibitor of apoptosis (XIAP) [23]. The E3 ubiquitin ligases activity of TRIM13, TRIM19, TRIM24 and TRIM28 regulate the stability or transcriptional activity of p53 that leads to the control of apoptosis and DNA damage response [24]. 
Finally TRIM19, TRIM25, and TRIM68, by regulating the activation of nuclear and hormone receptors, play a key role in the progression of leukaemia, as well as the development of breast and prostate cancer [21]. Based on this group of experimental data, we can hypothesize that defects in TRIM8 E3 ligase activity in glioma cells might promote carcinogenesis and cancerous growth by contributing to oncogenes stabilization and/or enhancing tumour suppressors degradation.

\section{Conclusions}

Our data give preliminary evidences that TRIM8 may participate in the carcinogenesis and progression of glioma and that the transcriptional repression of TRIM8 might have potential value for predicting poor prognosis in glioma patients. Moreover, our preliminary results suggest that TRIM8 and miR-17 may be part of a same circuit involved in glioma pathogenesis, although further experiments are needed to confirm the involvement of this modulation in gliomagenesis.

\section{Additional files}

Additional file 1: Table S1. Clinical-pathological patients' characteristics according to $\mathrm{WHO}$ grade classification.

Additional file 2: Table S2. Clinical-pathological patients' characteristics according to WHO grade classification (TCGA).

Additional file 3: Figure S3. TRIM8 expression level in transfected U87MG and GBM cells. mRNA and protein level of TRIM8 in U87MG (A) and GBM (B) cell lines transfected with a vector expressing TRIM8 or an empty vector was detected by $\mathrm{PPCR}(\mathrm{A}-\mathrm{B})$ and Western Blot (A-B), respectively, $72 \mathrm{~h}$ post transfection.

\section{Abbreviations}

GMB: Glioblastoma multiforme; TRIM: Tripartite motif-containing protein; CSS: Casa Sollievo della Sofferenza; NHA: Normal human astrocyte; QPCR: quantitative polymerase chain reaction; MTT: 3-2, 5-diphenyl tetrazolium bromide; TCGA: The Cancer Genome Atlas; LGG: Lower Grade Gliomas; PFS: Progression-free survival; OS: Overall Survival; HR: hazards ratio; CNV: Copy Number Variations; WHO: World Health Organization; ORF: Open Reading Frame; UTR: Untranslated Region.

\section{Competing interests}

The authors declare that they have no competing interests.

\section{Authors' contributions}

$L M$ and GM designed, directed the study, and drafted the manuscript. LM, $R B, P D, B M$ and $D C$ performed genetics and functional assays. $L M$ and $G M$ interpreted data. GM, PP, and VMF organized the recruitment of patients. LM, MTP, CF generated primary glioma cells. BA performed GPCRs and mutational screening. AD carried out the clonogenic assay. AF and $M C$ performed statistical analysis. VD collected specimens. LMCD pathologically classified all cases. CN and LL provided additional samples. All authors read and approved the final manuscript.

\section{Acknowledgements}

We are grateful to Mirko Fanelli, University of Urbino, for providing us with Normal Human Astrocytes. This work was supported by Associazione Italiana per la Ricerca sul Cancro (AIRC, IG \#14078), Ricerca Corrente 2012-14 granted by the Italian Ministry of Health, and the " $5 \times 1000$ " voluntary contributions to GM, Ricerca Finalizzata 2011 granted by the Italian Ministry of Health to LM.
The founders had no role in study design, data collection and analysis, decision to publish, or preparation of the manuscript.

\section{Author details}

'Medical Genetics Unit, IRCCS Casa Sollievo della Sofferenza, Poliambulatorio Giovanni Paolo II, I-71013 San Giovanni Rotondo (FG), Italy. ${ }^{2}$ Biostatistics Unit, IRCCS Casa Sollievo della Sofferenza, Poliambulatorio Giovanni Paolo II, I-71013 San Giovanni Rotondo (FG), Italy. ${ }^{3}$ Laboratory of Oncology, IRCCS Casa Sollievo della Sofferenza, I-71013 San Giovanni Rotondo (FG), Italy. ${ }^{4}$ Ph.D program in Experimental and Regenerative Medicine, University of Foggia, Foggia, Italy. ${ }^{5}$ Pathology Unit, IRCCS Casa Sollievo della Sofferenza, I-71013 San Giovanni Rotondo (FG), Italy. ${ }^{6}$ Neurosurgery Unit, IRCCS Casa Sollievo Della Sofferenza, San Giovanni Rotondo (FG), Italy. ${ }^{7}$ Medical Genetics, Department of Health Sciences, Università degli Studi di Milano, Milan, Italy. ${ }^{8}$ Laboratory of Medical Cytogenetics and Molecular Genetics, Istituto Auxologico Italiano, Milan, Italy. ${ }^{9}$ Gene Transfer Lab; IRCSS Azienda Ospedaliera Universitaria San Martino-IST Istituto Nazionale per la Ricerca sul Cancro, Genoa, Italy.

Received: 19 February 2015 Accepted: 19 May 2015

Published online: 16 June 2015

\section{References}

1. Marumoto T, Saya H. Molecular biology of glioma. Adv Exp Med Biol. 2012;746:2-11.

2. Ohgaki H, Dessen $P$, Jourde B, Horstmann S, Nishikawa T, Di Patre PL, et al. Genetic pathways to glioblastoma: a population-based study. Cancer Res. 2004;64(19):6892-9.

3. Furnari FB, Fenton T, Bachoo RM, Mukasa A, Stommel JM, Stegh A, et al. Malignant astrocytic glioma: genetics, biology, and paths to treatment. Genes Dev. 2007;21(21):2683-710.

4. Rasheed BK, McLendon RE, Friedman HS, Friedman AH, Fuchs HE, Bigner $\mathrm{DD}$, et al. Chromosome 10 deletion mapping in human gliomas: a common deletion region in 10q25. Oncogene. 1995;10(11):2243-6.

5. Reymond A, Meroni G, Fantozzi A, Merla G, Cairo S, Luzi L, et al. The tripartite motif family identifies cell compartments. EMBO J. 2001;20(9):2140-51.

6. Okumura F, Matsunaga Y, Katayama Y, Nakayama KI, Hatakeyama S. TRIM8 modulates STAT3 activity through negative regulation of PIAS3. J Cell Sci. 2010;123(Pt 13):2238-45.

7. Caratozzolo MF, Micale L, Turturo MG, Cornacchia S, Fusco C, Marzano F, et al. TRIM8 modulates p53 activity to dictate cell cycle arrest. Cell Cycle. 2012;11(3):511-23.

8. Carinci F, Arcelli D, Lo Muzio L, Francioso F, Valentini D, Evangelisti R, et al. Molecular classification of nodal metastasis in primary larynx squamous cell carcinoma. Transl Res. 2007;150(4):233-45.

9. Caratozzolo MF, Valletti A, Gigante M, Aiello I, Mastropasqua F, Marzano F, et al. TRIM8 anti-proliferative action against chemo-resistant renal cell carcinoma. Oncotarget. 2014;5(17):7446-57.

10. Malzkorn B, Wolter M, Liesenberg F, Grzendowski M, Stuhler K, Meyer HE, et al. Identification and functional characterization of microRNAs involved in the malignant progression of gliomas. Brain Pathol. 2011;20(3):539-50.

11. Bomben R, Gobessi S, Bo MD, Volinia S, Marconi D, Tissino E, Benedetti D, Zucchetto A, Rossi D, Gaidano $\mathrm{G}$ et al.: The miR-17 approximately 92 family regulates the response to toll-like receptor 9 triggering of CLL cells with unmutated IGHV genes. Leukemia 2012.

12. Stupp R, Hegi ME, Mason WP, van den Bent MJ, Taphoorn MJ, Janzer RC et al. Effects of radiotherapy with concomitant and adjuvant temozolomide versus radiotherapy alone on survival in glioblastoma in a randomised phase III study: 5-year analysis of the EORTC-NCIC trial. Lancet Oncol. 2009;10(5):459-66.

13. Roversi G, Pfundt R, Moroni RF, Magnani I, van Reijmersdal S, Pollo B, et al. Identification of novel genomic markers related to progression to glioblastoma through genomic profiling of 25 primary glioma cell lines. Oncogene. 2006;25(10):1571-83.

14. Rozen S, Skaletsky H. Primer3 on the WWW for general users and for biologist programmers. Methods Mol Biol. 2000;132:365-86.

15. Ferrero GB, Howald C, Micale L, Biamino E, Augello B, Fusco C, et al. An atypical 7q11.23 deletion in a normal IQ Williams-Beuren syndrome patient. Eur J Hum Genet. 2010;18(1):33-8. 
16. Howald C, Merla G, Digilio MC, Amenta S, Lyle R, Deutsch S, et al. Two high throughput technologies to detect segmental aneuploidies identify new Williams-Beuren syndrome patients with atypical deletions. J Med Genet. 2006;43(3):266-73.

17. Micale L, Augello B, Fusco C, Selicorni A, Loviglio MN, Silengo MC, et al. Mutation spectrum of MLL2 in a cohort of Kabuki syndrome patients. Orphanet J Rare Dis. 2011;6:38.

18. Carra E, Barbieri F, Marubbi D, Pattarozzi A, Favoni RE, Florio T, et al. Sorafenib selectively depletes human glioblastoma tumor-initiating cells from primary cultures. Cell Cycle. 2013;12(3):491-500.

19. Network. CGAR: Comprehensive genomic characterization defines human glioblastoma genes and core pathways. Nature. 2008:455(7216):1061-8.

20. Lu S, Wang S, Geng S, Ma S, Liang Z, Jiao B. Increased expression of microRNA-17 predicts poor prognosis in human glioma. J Biomed Biotechnol. 2012;2012:970761.

21. Hatakeyama S. TRIM proteins and cancer. Nat Rev Cancer. 2011;11(11):792-804.

22. Micale L, Chaignat E, Fusco C, Reymond A, Merla G. The tripartite motif: structure and function. Adv Exp Med Biol. 2012;770:11-25.

23. Ryu YS, Lee Y, Lee KW, Hwang CY, Maeng JS, Kim JH, et al. TRIM32 protein sensitizes cells to tumor necrosis factor (TNFalpha)-induced apoptosis via its RING domain-dependent E3 ligase activity against X-linked inhibitor of apoptosis (XIAP). J Biol Chem. 2011;286(29):25729-38.

24. Hock A, Vousden KH. Regulation of the p53 pathway by ubiquitin and related proteins. Int J Biochem Cell Biol. 2010;42(10):1618-21.

\section{Submit your next manuscript to BioMed Central and take full advantage of:}

- Convenient online submission

- Thorough peer review

- No space constraints or color figure charges

- Immediate publication on acceptance

- Inclusion in PubMed, CAS, Scopus and Google Scholar

- Research which is freely available for redistribution 